\title{
MD Investigações: um jogo educacional aberto para auxiliar a aprendizagem de lógica
}

\author{
Carlos Magno ${ }^{1}$, Lucas Felix ${ }^{1}$, Lucas Lagoa ${ }^{1}$, Leonardo Rocha ${ }^{1}$, \\ Elisa Albergaria ${ }^{1,2}$ \\ ${ }^{1}$ Departamento de Ciência da Computação - Universidade Federal de São João del Rei \\ UFSJ - MG \\ ${ }^{2}$ Núcleo de Educação a Distância - NEAD - Universidade Federal de São João del Rei \\ $\mathrm{UFSJ}-\mathrm{MG}$ \\ carlosmagno@outlook.com, lucasgsfelix@gmail.com, \\ lucaslagoanogueira@hotmail.com, lcrocha@ufsj.edu.br \\ elisa@nead.ufsj.edu.br
}

\begin{abstract}
This article presents the "MD Investigations", an educational game to aid in the learning process of logic and syllogism, subject of several courses in Computer Science, especially in Discrete Mathematics, whose retention rate is high. The MD Investigações is an Open Educational Resource (OER), where the story of the game is simple and thought-provoking, using the playful theme of investigations. The story develops from the perspective of a student who had his teacher kidnapped and, to be able to find him, needs to solve a series of puzzles using logical reasoning. The game development process is the "Design", proposed in the Human Computer Interaction context. The target audience are the students in the beginning of the Computer Science course, where Discrete Mathematics is a common subject.
\end{abstract}

Resumo. Este artigo apresenta o MD Investigações, um jogo educacional para auxiliar no processo de aprendizagem de lógica e silogismo, tema de diversas disciplinas de cursos de Ciência da Computação, em especial Matemática Discreta, cujo índice de retenção é alto. O MD Investigações é um Recurso Educacional Aberto (REA), onde a história do jogo é simples e instigante, utilizando o tema lúdico de investigações. A história se desenvolve sob a perspectiva de um aluno que teve seu professor raptado e precisa resolver uma série de quebra-cabeças utilizando raciocínio lógico, para poder encontrá-lo. O processo de desenvolvimento do jogo foi o design, proposto dentro do contexto da Interação Humano Computador. O público alvo são os alunos dos primeiros períodos do curso de Ciência da Computação, onde normalmente a disciplina de Matemática Discreta é ministrada.

Agradecimentos: $O$ presente trabalho foi realizado com apoio da Coordenação de Aperfeiçoamento de Pessoal de Nível Superior - Brasil (CAPES) - Código de Financiamento 001, NEAD/UFSJ, CNPq e FAPEMIG. 
VII Congresso Brasileiro de Informática na Educação (CBIE 2018)

Anais do XXIX Simpósio Brasileiro de Informática na Educação (SBIE 2018)

\section{Introdução}

Segundo levantamento feito pela Organização para a Cooperação e Desenvolvimento Econômico ${ }^{1}$ realizado em $2016,14 \%$ da população brasileira possuía o ensino superior, sendo esta uma margem baixa perante a média de $35 \%$ dos demais países avaliados pela organização. Entretanto, entre 2006 e 2016, as matrículas na educação superior aumentaram 62,8\%, com uma média anual de 5\% de crescimento [INEP, 2016]. Ainda segundo o Censo da Educação Superior de 2016, o número de matrículas na modalidade a distância cresceu ainda mais, atingindo quase 1,5 milhão em 2016, o que representava uma participação de $18,6 \%$ do total de matrículas da educação superior.

$\mathrm{O}$ crescimento da modalidade de ensino a distância resulta em um impacto direto sobre a iniciativa pública e privada que passam a ser pressionadas a atender uma crescente demanda por um modelo de educacional dinâmico, colaborativo, descentralizado, flexível [Okada, 2013]. Dentre as formas que surgiram em decorrência da procura de novas maneiras de aprendizado temos os Recursos Educacionais Abertos (REAs). Um REA pode ser qualquer material destinado ao ensino, pesquisa e aprendizado que esteja sob domínio público ou licenciado de maneira aberta, permitindo que os mesmos sejam adaptados e utilizados por terceiros [UNESCO, 2002].

Apesar de originalmente propostos para o cenário de ensino a distância, atualmente os REAs são utilizados nos mais diferentes contextos de ensino, abordando conteúdos de várias áreas, tanto a distância quanto presencial. REAs são verdadeiras contribuições que as experiências de ensino a distância trouxeram para o ensino presencial. Um exemplo é apresentado por [Amiel, 2014] que em seu trabalho busca analisar o papel dos recursos educacionais no ensino básico, com enfoque na área de história. Outro bom exemplo do alcance que REAs tem alcançado é a utilização dos mesmos como suporte também no ensino superior, onde os alunos buscam aprofundar mais nos conteúdos e a variedade de tipos de recursos é vista como uma possibilidade de realizar esse aprofundamento.

Os REAs têm sido propostos para diferentes áreas do ensino superior com o objetivo de reduzir altas taxas de evasão. O problema de evasão universitária tem gerado prejuízos financeiros, sociais e acadêmicos, tanto para as Instituições Federais de Ensino Superior (IFES) quanto para os alunos [Da Cunha et al. 2016]. Na lista dos cursos de maior evasão em todo mundo estão àqueles relacionados a ciências exatas, tais como Ciência da Computação, Matemática e as Engenharias. Dessa forma, iniciativas que visam ampliar a possibilidade de aprendizagem nesses cursos são essenciais.

Assim, nesse contexto, este trabalho apresenta um REA na forma de um jogo que auxilia no processo de desenvolvimento de raciocínio lógico, inserido no contexto da disciplina de Matemática Discreta, normalmente ministrada nos primeiros períodos do curso de Ciência da Computação. Trata-se de uma disciplina do ciclo básico, fundamental para as diversas subáreas da computação, que apresenta um alto índice de retenção. O jogo utiliza uma temática lúdica apresentando diversos problemas que precisam ser resolvidos utilizando lógica e silogismo, cujo objetivo principal é criar

\footnotetext{
${ }^{1}$ http://www.oecd.org/
} 
VII Congresso Brasileiro de Informática na Educação (CBIE 2018)

Anais do XXIX Simpósio Brasileiro de Informática na Educação (SBIE 2018)

mecanismos para que os alunos possam estudar, aplicando os conhecimentos adquiridos em sala de aula.

\section{Trabalhos Relacionados}

Atualmente, a criação e uso de recursos no contexto da educação é tema recorrente, existindo diversos trabalhos que visam o aprimoramento do aprendizado em diferentes áreas. Os Recursos Educacionais Abertos, também conhecidos como REAs, são exemplos desses recursos, pois tem como objetivo facilitar e disseminar o conhecimento. Formalmente, REAs "são materiais para ensinar, aprender e pesquisar que estão em domínio público ou são publicados com licença de propriedade intelectual que permite sua livre utilização, adaptação e distribuição." [UNESCO, 2012].

Vários são os trabalhos na literatura que avaliam, utilizam e estudam a abrangência dos REAs. O trabalho Costa et al (2016), por exemplo, avalia o estado da arte de recursos educacionais empregados no ensino e aprendizado da linguística, mostrando que existem poucos REAs voltados para o estudo de línguas no Brasil. Na mesma linha, o artigo de [Leffa, 2016] também avalia a utilização de REAs no ensino de línguas, entretanto, este avalia a evolução dos chamados objetos de aprendizagem que são definidos no texto como tipo de aprendizagem em que se utiliza computadores priorizando a utilização de componentes reutilizáveis até os atuais recursos educacionais abertos [Wiley, 2000]. Apesar da importância dos REAs, estudos recentes [Hilu et al, 2015] apontam que os programas de pós-graduação, que além de pesquisadores visam formar docentes, não colocam seus alunos em contato com esses recursos, consequentemente formando futuros docentes que não estão aptos a disponibilizarem seus materiais de forma aberta e gratuita.

Por outro lado, jogos digitais são hoje extremamente populares e talvez uma das principais fontes de diversão de crianças, adolescentes e até mesmo adultos. Consequentemente, uma linha de trabalhos que vem ganhando destaque recentemente é a adoção de jogos digitais como REAs (também chamados de jogos sérios). Diversos são os estudos que visam a utilização dos mesmos como formas lúdicas de aprendizado [Fernandes, 2016], [Oliveira et al, 2015], [Martins et al, 2016]. Em seu trabalho, Fernandes (2016) além de descrever a utilização de jogos digitais como ferramenta no auxílio da aprendizagem, enfatiza a contribuição dos jogos no desenvolvimento das percepções, inteligência e socialização de crianças. Já Oliveira et al (2015) faz uma avaliação de jogos educacionais na área de matemática, mostrando o quanto este tipo de processo é importante para validar a competência dos jogos diante de seus objetivos. Martins et al, (2016) apresenta um relato de experiência do uso do jogo Labirinto Clássico, disponível na plataforma Code.org ${ }^{2}$, que foi avaliado por 168 alunos do ensino fundamental nos quesitos de interação, diversão e aprendizado.

$\mathrm{O}$ uso de jogos educacionais pode ser aplicado nos mais variados contextos e públicos de diferentes graus de escolaridade. Assim, pode-se utilizar um jogo para auxiliar no processo de alfabetização de crianças, assim como para alunos em uma disciplina de um curso de graduação. Dentre as inúmeras possibilidades, podemos ressaltar os conteúdos dos cursos da área de ciências exatas, visto que a dificuldade dos

\footnotetext{
${ }^{2}$ https://code.org/
} 
VII Congresso Brasileiro de Informática na Educação (CBIE 2018)

Anais do XXIX Simpósio Brasileiro de Informática na Educação (SBIE 2018)

alunos nessa área costuma ser um fator relevante a ser trabalhado. Conteúdos de programação, cálculo, estatística, lógica, por exemplo, são densos e outros tipos de recursos podem auxiliar no processo de aprendizagem. $\mathrm{O}$ desenvolvimento do raciocínio lógico em jogos digitais, por exemplo, já foi foco nos seguintes trabalhos [Barbosa et al, 2017], [Kologeski et al, 2016], [Tonéis et al, 2015]. Os artigos [Barbosa et al, 2017] [Kologeski et al, 2016] objetivam o ensino da lógica utilizando tecnologias da informação desenvolvendo um jogo e aplicando o mesmo em alunos do ensino fundamental. Já o estudo de [Tonéis et al, 2015] propõe um jogo protótipo dando enfoque no desenvolvimento do raciocínio lógico e matemático. $O$ jogo aqui apresentado tem como diferencial ter sido desenvolvido em código aberto e estar disponível para que qualquer pessoa possa utilizar ou modificar ${ }^{3}$. Outra vantagem é que MD Investigações é multiplataforma e pode ser utilizado localmente, desde que instalado no dispositivo, sejam de computadores até celulares ou tablets. Além disso, o público alvo do jogo envolve o ensino superior, com questões que abordam um raciocínio mais profundo sobre o tema. Ele não se limita a alunos do curso de Ciência da Computação, mas interessados em jogos que envolvem raciocínio lógico de uma forma geral, tendo uma maior abrangência.

\section{Metodologia}

As etapas de desenvolvimento desse trabalho são apresentadas descrevendo os passos seguidos no desenvolvimento do jogo MD Investigações. As atividades realizadas seguiram o processo de design [Lawson, 2006] no contexto da Interação Humano Computador [Preece, 1994]. O termo Interação Humano-Computador (IHC) é utilizado para descrever um novo campo de estudo e que abrange não apenas interfaces, mas todos os aspectos relacionados à interação entre pessoas e sistemas computacionais. Por sua vez, um processo de design, em linhas gerais, envolve atividades de (1) análise, (2) síntese e (3) avaliação. A análise busca estudar e interpretar a situação atual, a síntese consiste em planejar e executar uma intervenção na situação e, por fim, a avaliação consiste em avaliar o efeito da intervenção, verificando se ficou conforme desejado.

No contexto da análise, o primeiro passo consistiu na criação e divulgação de um questionário respondido pelos alunos do primeiro período do curso de Ciência da Computação da Universidade Federal de São João Del Rei que cursavam a disciplina de Matemática Discreta. As perguntas elaboradas abordaram temas sobre o histórico dos usuários (conhecimento prévio antes de iniciar o curso), dificuldades existentes durante o primeiro período (quais disciplinas e conteúdos são considerados mais difíceis) e hábitos de estudos (recursos mais utilizados e frequência de estudo).

O segundo passo consistiu na criação de personas. Persona é uma técnica de usabilidade que consiste na criação de perfis e personificação de grupo de usuários, ou seja, representa uma caracterização de um personagem que, embora seja fictício, expõe as características importantes da população de usuários para a qual se destina o produto o projeto [Adlin 2006]. Juntamente com as personas, foram criados cenários de uso, ilustrando o contexto onde o REA, no caso o jogo digital MD Investigações, seria aplicado e utilizado.

\footnotetext{
${ }^{3}$ Código disponível em https://github.com/lucaslagoa/REA_IHC/releases
} 
VII Congresso Brasileiro de Informática na Educação (CBIE 2018)

Anais do XXIX Simpósio Brasileiro de Informática na Educação (SBIE 2018)

$\mathrm{Na}$ etapa de síntese, protótipos foram elaborados, desde baixa fidelidade até protótipos funcionais de alta fidelidade que foram apresentados aos usuários. Por fim, para o jogo ser validado como uma ferramenta de ensino lúdico válida para o aprendizado, o mesmo foi analisado por professores que ministram ou já ministraram as disciplinas "Matemática Discreta" e "Lógica Aplicada à Computação" do curso de Ciência da Computação da UFSJ. Para isso, esses professores responderam um questionário visando a avaliação do jogo como meio de instrução. Uma análise também foi feita com alunos da disciplina para avaliar a interface do jogo sob a visão do jogador (o aluno), verificando se a mesma está simples e apta a alcançar seu objetivo, que é auxiliar no desenvolvimento do pensamento lógico.

\section{O jogo MD Investigações}

Nessa seção apresentamos o "MD Investigações", detalhando a história do jogo, o processo de desenvolvimento do mesmo e descrevendo também a validação realizada com potenciais usuários e envolvidos no processo de ensino aprendizagem. $\mathrm{O}$ jogo foi desenvolvido pensando em seu público-alvo, alunos de graduação de Ciência da Computação, que cursavam a disciplina de Matemática Discreta, bem como outras disciplinas que também necessitam de conhecimentos de lógica e silogismo.

\subsection{O Jogo}

O REA desenvolvido se destaca por possuir interface simples e atrativa, visando se tornar, de fato, uma ferramenta de aprendizado que mescle estudo e diversão. Conforme mencionado anteriormente, o "MD Investigações" é um jogo digital que visa auxiliar o ensino de conteúdos relacionados à lógica e silogismo, fundamentos básicos no curso de Ciência da Computação, sendo o tema principal da disciplina de Matemática Discreta. Trata-se de uma disciplina obrigatória em quase todos os cursos de computação, fundamental para as diversas subáreas da computação e normalmente ministrada nos primeiros períodos do curso e com alto índice de retenção.

O "MD Investigações" foi desenvolvido utilizando a plataforma de desenvolvimento de games livre, Godot ${ }^{4}$, sendo esta uma ferramenta livre e aberta. As primeiras telas do jogo apresentam o modo como jogar, assim como informações que apresentam o mesmo, como é possível visualizar na Figura 1.

A história do jogo é simples e instigante para os usuários por utilizar o tema lúdico de investigações, onde pequenos problemas precisam ser resolvidos para que uma determinada investigação seja concluída. A história se desenvolve sob a perspectiva de um aluno que teve seu professor raptado e precisa resolver uma série de quebra-cabeças para poder encontrar seu instrutor. Além do propósito geral que é o desenvolvimento do raciocínio lógico do jogador, o jogo ainda estimula a criatividade e traz ao usuário a oportunidade de aprender a sua maneira e ao seu tempo, diferentemente das estratégias tradicionais, as quais podem ser desestimulantes e maçantes.

\footnotetext{
${ }^{4}$ https://godotengine.org/
} 
VII Congresso Brasileiro de Informática na Educação (CBIE 2018)

Anais do XXIX Simpósio Brasileiro de Informática na Educação (SBIE 2018)
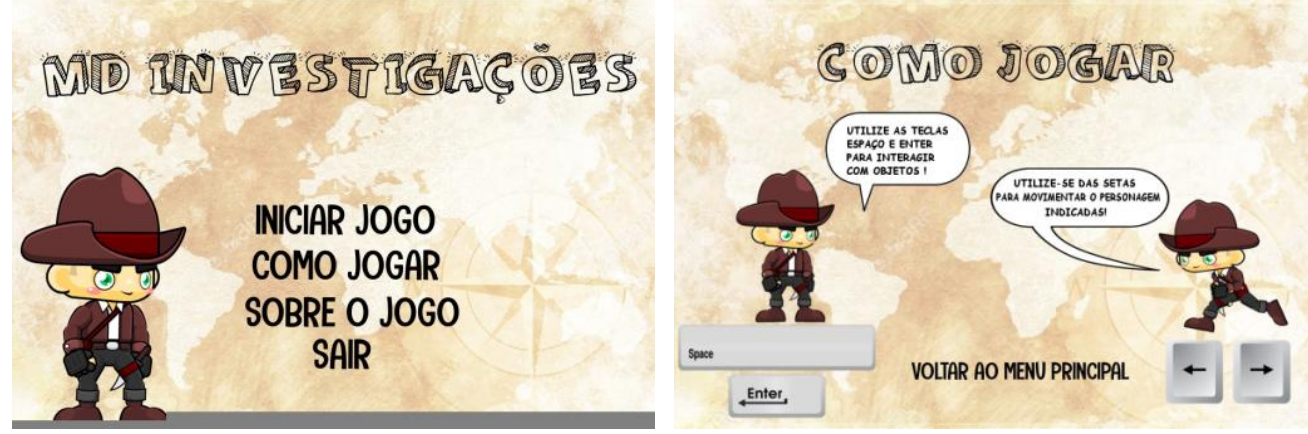

Figura 1. Telas iniciais do jogo MD Investigações

Atualmente o jogo está dividido em três fases: "Floresta", "Cemitério" e "Deserto", cada parte possuindo uma história e objetos contextualizados, sendo ao final da fase mostrado ao usuário um diário com todas as pistas que o mesmo coletou durante a fase. O propósito de cada fase é recolher as pistas, interagindo com os objetos e utilizando premissas que auxiliam o jogador a responder uma questão final de lógica e, consequentemente, passar de fase. Na Figura 2 é possível visualizar cenários diferentes do jogo de acordo com a fase e a Figura 3 mostra uma questão a ser respondida pelo usuário, de modo que ele avance no jogo.

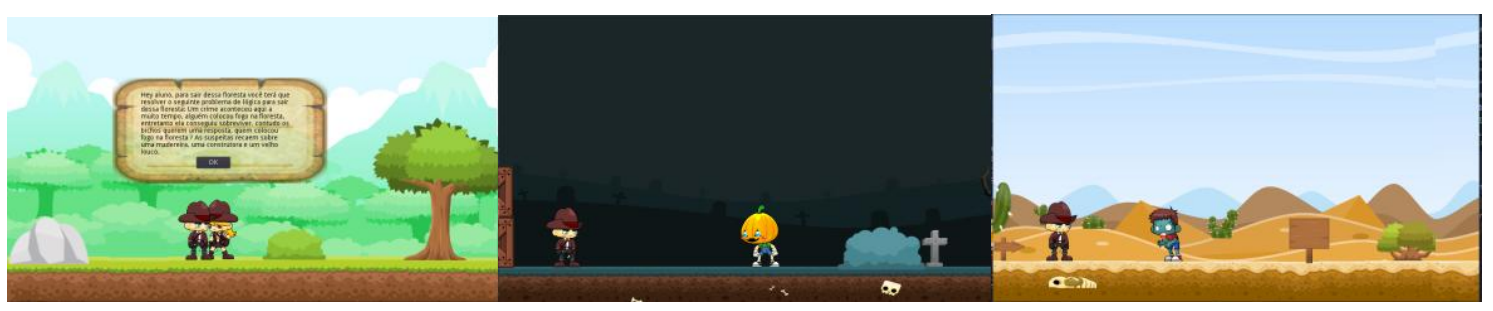

Figura 2. Diferentes cenários apresentados no Jogo: Floresta, Cemitério e Deserto

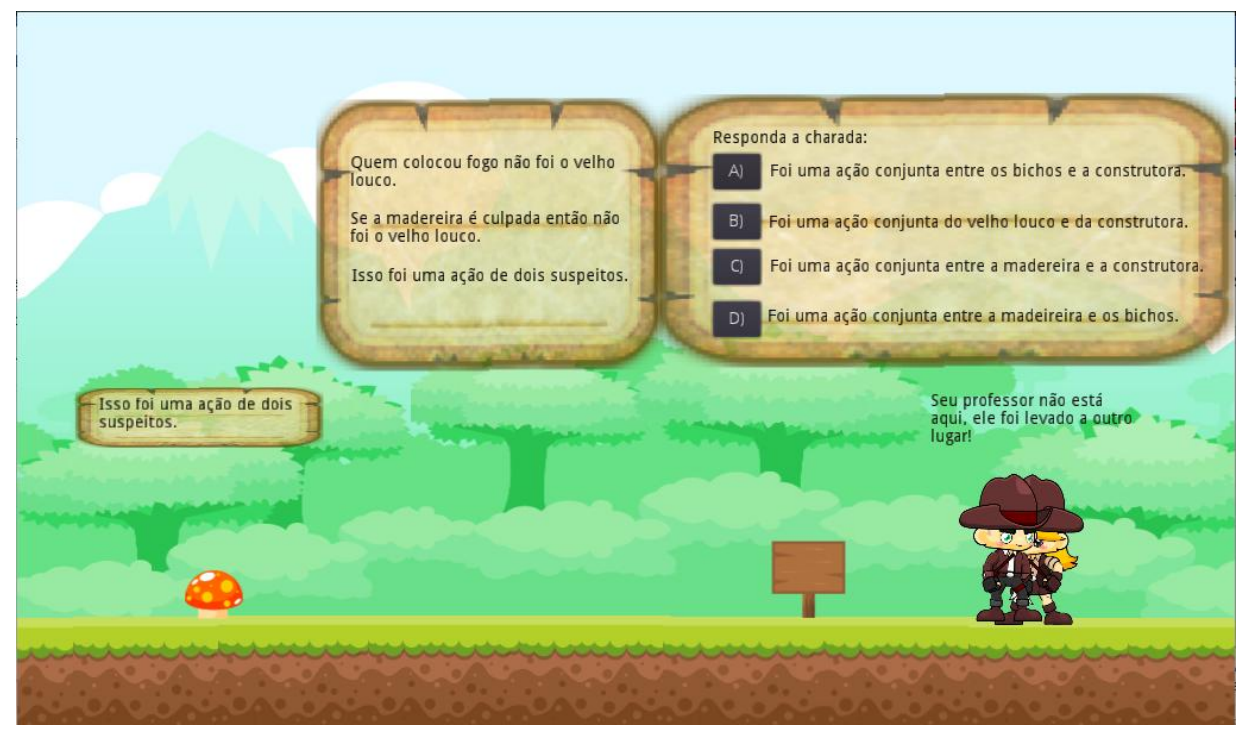

Figura 3. Tela do Jogo MD Investigações apresentando uma questão de lógica a ser respondida baseada nas pistas obtidas 
VII Congresso Brasileiro de Informática na Educação (CBIE 2018)

Anais do XXIX Simpósio Brasileiro de Informática na Educação (SBIE 2018)

\subsection{Validação do Jogo}

Para avaliarmos o REA desenvolvido, após a implementação do jogo “MD Investigações", foi feita uma validação com alunos dos primeiros períodos do curso de Ciência da Computação e entrevistas com docentes que lecionam disciplinas relacionadas ao conteúdo de lógica, tais como "Matemática Discreta" e "Lógica Aplicada à Computação". Esta validação com os professores foi baseada no Método de Explicitação do Discurso Subjacente (MEDS), um método qualitativo de pesquisa desenvolvido por Nicolaci-da-Costa [Nicolaci-da-Costa, 2007]. A primeira etapa foi definir o objetivo da validação, sendo analisar a ferramenta como um método lúdico de ensino, que possa dar auxílio a professores e alunos como modo de ensino e aprendizagem.

A escolha dos entrevistados foi feita baseada nas disciplinas lecionadas pelos mesmos. Após a apresentação presencial do jogo aos professores foi aplicado um questionário com cinco perguntas para avaliação qualitativa do REA. De forma a apresentar os dados coletados, na tabela abaixo podemos visualizar as perguntas realizadas e as respostas obtidas:

\begin{tabular}{|c|c|c|c|c|}
\hline Questões: & Professor \#1 & Professor \#2 & Professor \#3 & Professor \#4 \\
\hline $\begin{array}{c}\text { Você acha que os } \\
\text { alunos conseguem } \\
\text { se apropriar da } \\
\text { matéria apenas } \\
\text { com o conteúdo } \\
\text { passado em sala } \\
\text { de aula? }\end{array}$ & $\begin{array}{l}\text { "Nem sempre, } \\
\text { existem } \\
\text { conteúdos que } \\
\text { precisam de } \\
\text { tempo e empenho } \\
\text { dos alunos" }\end{array}$ & $\begin{array}{c}\text { "Não, eles tem a } \\
\text { necessidade de buscar } \\
\text { algo além e estudar } \\
\text { em casa" }\end{array}$ & "Não" & $\begin{array}{l}\text { “Não. Tudo que é } \\
\text { passado em aula deve } \\
\text { ser reforçado por meio } \\
\text { de exercícios extra- } \\
\text { sala, monitorias ou } \\
\text { qualquer outra solução } \\
\text { que tenha o intuito de } \\
\text { auxiliar o processo } \\
\text { ensino-aprendizagem. } \\
\text { No caso, o jogo de } \\
\text { vocês faz esse papel" }\end{array}$ \\
\hline $\begin{array}{c}\text { Você acha que } \\
\text { formas diferentes } \\
\text { das tradicionais } \\
\text { podem auxiliar no } \\
\text { aprendizado do } \\
\text { aluno? }\end{array}$ & $\begin{array}{c}\text { "Sim, é } \\
\text { importante haver } \\
\text { maneiras de } \\
\text { despertar o } \\
\text { interesse dos } \\
\text { alunos pelos } \\
\text { diversos } \\
\text { conteúdos" }\end{array}$ & $\begin{array}{l}\text { "Sim, a geração que } \\
\text { está na faculdade não } \\
\text { aprende apenas com } \\
\text { os métodos } \\
\text { tradicionais" }\end{array}$ & $\begin{array}{c}\text { "Ferramentas/Rec } \\
\text { ursos visuais em } \\
\text { geral: animações, } \\
\text { esquemas, jogos, } \\
\text { etc" }\end{array}$ & $\begin{array}{l}\text { "Sim, o uso de jogos } \\
\text { pode auxiliar no } \\
\text { aprendizado, uma vez } \\
\text { que o público-alvo é } \\
\text { consumidor de jogos" }\end{array}$ \\
\hline $\begin{array}{c}\text { Você crê que o } \\
\text { jogo “MD } \\
\text { Investigações" } \\
\text { pode auxiliar no } \\
\text { aprendizado dos } \\
\text { alunos de alguma } \\
\text { forma? }\end{array}$ & $\begin{array}{c}\text { "Sim, } \\
\text { principalmente } \\
\text { para alunos que } \\
\text { gostam de jogos } \\
\text { eletrônicos" }\end{array}$ & $\begin{array}{l}\text { "Sim, foi muito bem } \\
\text { desenvolvido" }\end{array}$ & $\begin{array}{c}\text { "Sim, toda } \\
\text { ferramenta lúdica } \\
\text { devidamente } \\
\text { direcionada tem o } \\
\text { potencial de } \\
\text { auxiliar no } \\
\text { aprendizado" }\end{array}$ & "Sim" \\
\hline Se você pudesse & "Criar uma & "Eu apenas & "Acrescentar mais & "No caso, pensando na \\
\hline
\end{tabular}


VII Congresso Brasileiro de Informática na Educação (CBIE 2018)

Anais do XXIX Simpósio Brasileiro de Informática na Educação (SBIE 2018)

\begin{tabular}{|c|c|c|c|c|}
\hline $\begin{array}{c}\text { melhorar o jogo } \\
\text { de alguma forma } \\
\text { (adicionar, } \\
\text { remover itens) } \\
\text { como você iria } \\
\text { melhorar o mesmo } \\
?\end{array}$ & \begin{tabular}{|c|} 
interface que \\
permita ao \\
professor inserir \\
novos problemas. \\
Avisar ao aluno \\
sobre a existência \\
de pistas falsas"
\end{tabular} & $\begin{array}{c}\text { aumentaria o número } \\
\text { de pistas para usar } \\
\text { inferência lógica" }\end{array}$ & $\begin{array}{c}\text { fases e exibiria, a } \\
\text { cada alternativa } \\
\text { marcada } \\
\text { incorretamente, } \\
\text { uma breve } \\
\text { explicação ou dica } \\
\text { do porquê daquela } \\
\text { alternativa não ser } \\
\text { correta (sem } \\
\text { entregar a } \\
\text { resposta)" }\end{array}$ & $\begin{array}{l}\text { questão de deduções, } \\
\text { que é o que o jogo } \\
\text { possui até o momento, } \\
\text { necessita acrescentar } \\
\text { alguns passos para } \\
\text { resolução das questões } \\
\text {, como por exemplo: } \\
\text { definição de } \\
\text { argumentos, } \\
\text { proposições, tradução } \\
\text { dos fatos para as } \\
\text { proposições e o passo- } \\
\text { a-passo das deduções" }\end{array}$ \\
\hline \begin{tabular}{|} 
Qual sua opinião \\
quanto a \\
dificuldade da \\
resolução dos \\
problemas do jogo \\
$?$
\end{tabular} & \begin{tabular}{|c|} 
"Para alunos \\
iniciantes $\left(1^{\circ}\right.$ \\
período), os \\
problemas devem \\
ser simples nas \\
$1^{\text {a }}$ s fases"
\end{tabular} & $\begin{array}{c}\text { "Um aumento no } \\
\text { número de pistas } \\
\text { aumentaria a } \\
\text { dificuldade do jogo" }\end{array}$ & $\begin{array}{l}\text { "Adequada para } \\
\text { alunos de } \\
\text { graduação", }\end{array}$ & $\begin{array}{c}\text { "A dificuldade está } \\
\text { excelente" }\end{array}$ \\
\hline
\end{tabular}

Tabela 1: Respostas dos professores ao questionário proposto

A partir da tabela, é possível observar que as respostas apresentadas pelos docentes avaliados convergem. Analisando detalhadamente as mesmas, é possível perceber que todos educadores concordam que não é possível que os alunos se apropriem totalmente dos conceitos apenas com o conteúdo passado em sala de aula. Todos estão de acordo que ferramentas como "MD Investigações" podem auxiliar bastante tanto educadores quanto alunos, estes últimos de forma a propiciar liberdade de escolha de aprendizado, já que desta forma são disponibilizadas novas formas para se aprender o conteúdo.

Como sugestões de novas funcionalidades, os docentes apresentaram ideias para a evolução do trabalho como um todo. Na parte didática, a sugestão foi de acrescentar novos problemas, mais fases e mais conteúdos relacionados a matérias. Em relação a interface, foram dadas sugestões em relação a exibição de opções incorretas marcadas e uma pequena explicação do porquê a mesma está errada. De toda forma, vale destacar ao final que o jogo foi bem aceito pelos professores, sendo o mesmo avaliado como uma ferramenta que pode contribuir no ensino das disciplinas lecionadas por estes.

Para validar de maneira mais consistente O REA proposto, o jogo foi apresentado ao público-alvo (alunos do primeiro período que estavam cursando a disciplina de Matemática Discreta). A eles foram dadas três tarefas que corroboram na robustez do jogo. É importante mencionar que tal validação foi feita seguindo padrões de teste de usabilidade, não havendo a interferência de avaliadores durante o processo. Abaixo, podemos ver as tarefas dadas aos usuários e as respostas obtidas na avaliação.

\begin{tabular}{|c|c|c|c|}
\hline Tarefas: & Usuário \#1 & Usuário \#2 & Usuário \#3 \\
\hline $\begin{array}{c}\text { Descobrir como interagir } \\
\text { com os objetos }\end{array}$ & Realizada com sucesso & Realizada com sucesso & Realizada com sucesso \\
\hline
\end{tabular}


VII Congresso Brasileiro de Informática na Educação (CBIE 2018)

Anais do XXIX Simpósio Brasileiro de Informática na Educação (SBIE 2018)

\begin{tabular}{|c|c|c|c|}
$\begin{array}{c}\text { O que está escrito na } \\
\text { segunda pista da fase }\end{array}$ & $\begin{array}{c}\text { Usuário não conclui a } \\
\text { tarefa, pois não achou } \\
\text { todas as pistas }\end{array}$ & Realizada com sucesso & Realizada com sucesso \\
\hline $\begin{array}{c}\text { Interagir com o } \\
\text { questionário final }\end{array}$ & Realizada com sucesso & Realizada com sucesso & Realizada com sucesso \\
\hline
\end{tabular}

Tabela: Mostra o resultado da interação dos usuários com jogos

A partir da tabela acima, é possível visualizar que maioria dos usuários conseguiu cumprir suas tarefas, ressaltando assim que a ferramenta possui uma interface intuitiva e de simples de interação. Entretanto, pode-se afirmar também que a ferramenta ainda possui pequenas melhoras a serem feitas já que nem todas as tarefas foram concluídas pela totalidade dos avaliadores. O problema encontrado pelo usuário \#1 ao executar a tarefa "O que está escrito na segunda pista da fase" foi a falta de coleta de todas as pistas presentes, pois o mesmo não interagiu com todos os objetos disponíveis. Isto evidenciou que os objetos de interação com usuário não se mostravam nitidamente diferenciáveis do resto do cenário. Além das sugestões apresentadas pelos docentes, ficou claro que precisamos evidenciar melhor os objetos de iteração do jogo.

\section{Considerações Finais}

Neste trabalho é proposto um jogo que oferta a usuários, alunos de graduação, uma maneira lúdica e interessante que possui como objetivo o desenvolvimento do pensamento lógico. Para o desenvolvimento deste foi utilizado a ferramenta aberta Godot e assim como esta, o jogo é totalmente aberto, sendo "MD Investigações" um recurso educacional aberto (REA). A validação da ferramenta foi feita por docentes e discentes do curso de ciência da computação da Universidade Federal de São João del Rei. Professores avaliaram a ferramenta no âmbito didático e alunos avaliaram a interface da ferramenta, sendo em ambos a resposta obtida majoritariamente positiva. Por fim, como trabalhos futuros visamos o desenvolvimento de novas fases e a utilização de ideias dadas pelos professores, para que a ferramenta possa ser no efetivamente utilizada durante em disciplinas como matemática discreta, lógica computacional, dentre outras.

\section{Referências Bibliográficas}

Adlin, Tamara \& Pruitt, John. (2006) The Persona Lifecycle: Keeping People in Mind Throughout Product Design. Morgan Kaufmann.

Amiel, Tel. (2014) Recursos Educacionais Abertos: uma análise a partir do livro didático de história.

Barbosa, D. N. et al. (2017) Ensinando lógica com as tecnologias da informação: Desenvolvendo o raciocínio lógico e o pensamento computacional. Cataventos Revista de extensão da Universidade de Cruz Alta, v. 9, n. 1, p. 54-72, 2017.

Costa, A. R. et al. (2016) Contribuindo com o estado da arte sobre Recursos Educacionais Abertos para o ensino e a aprendizagem de línguas no Brasil. Revista Veredas, v. 20, n. 1. 
VII Congresso Brasileiro de Informática na Educação (CBIE 2018)

Anais do XXIX Simpósio Brasileiro de Informática na Educação (SBIE 2018)

Da Cunha, J. V. A., Nascimento, E. M., and de Oliveira Durso, S. (2016). Razões e influências para a evasão universitária: um estudo com estudantes ingressantes nos cursos de ciências contábeis de instituições públicas federais da região sudeste. Advances in Scientific and Applied Accounting, 9(2):141-161.

Fernandes, J. C. L. (2016) Educação digital: Utilização dos jogos de computador como ferramenta de auxílio à aprendizagem. FaSCI-Tech, v. 1, n. 3

Hilu, L.; Lupion T., P; Behrens, M. (2015) REA (Recursos Educacionais Abertos) conhecimentos e (des) conhecimentos. Revista e-Curriculum, v. 13, n. 1.

INEP. Censo da Educação Superior 2016 - Notas Estatísticas. Instituto Nacional de Estudos e Pesquisas Educacionais Anísio Teixeira (INEP). Ministério da Educação (MEC), 2016.

Kologeski, Analise L; et al. (2016) Desenvolvendo o Raciocínio lógico e pensamento computacional: Experiências no Contexto do Projeto Logicando. RENOTE, v. 14, n. 2.

Lawson, B.R. (2006) How Designers Think: The Design Process Demystified, Fourth Edition. Architectural Press.

Leffa, Vilson J. (2016) Uma outra aprendizagem é possível : colaboração em massa, recursos educacionais abertos e ensino de línguas. Trabalhos em Linguística Aplicada, v. 55, n. 2, p. 353-377

Martins, R.; Reis, Ronaldo; Marques, A. B. (2016) Inserção da programação no ensino fundamental: Uma análise do jogo labirinto clássico da code.org através de um modelo de avaliação de jogos educacionais: In: Anais do Workshop de Informática na Escola, p. 121.

Nicolaci-da-Costa, A. M. (2007). O campo da pesquisa qualitativa e o Método de Explicitação do Discurso Subjacente (MEDS). Psicologia: Reflexão e Crítica, 20(1), 65-73.

Okada, A. (2013). Recursos Educacionais Abertos e Redes Sociais. São Luís: EDUEMA

Oliveira, Wilk et al. (2015) Avaliação de jogos educativos: uma abordagem no ensino de matemática. In: Brazilian Symposium on Computers in Education (Simpósio Brasileiro de Informática na Educação - SBIE). p. 657

Preece, Jenny; Fairbanks, Rollin; Hettinger, Zachary; Benda, Natalie. (1994) Humancomputer interaction. Addison-Wesley Longman Ltda.

Tonéis, Cristianl, Natal; F, Janete B. (2015) Os jogos digitais como espaço para produção de conhecimentos: O Raciocínio lógico e matemático em jogo. REU - Revista de Estudos Universitários, v. 41, n. 1, p. 25-39.

UNESCO - (2012) Organização das nações unidas para Educação, Ciência e Cultura. Recursos Educacionais Abertos (REA).

Wiley, D. A. (2000) .Learning object design and sequencing theory . Doctoral Dissertation, Brigham Young University. 